\title{
SMART Technology and Orthopedic Treatment and Rehabilitation
}

\author{
Thomas W Miller* \\ Department of Gerontology, College of Public Health, \& Department of Psychiatry, College Medicine, USA \\ *Corresponding author: Thomas W Miller, Professor Emeritus \& Senior Research Scientist, Institute for Health, Intervention \& \\ Policy, Department of Gerontology, College of Public Health, \& Department of Psychiatry, College Medicine, USA
}

\section{Mini Review}

There is a growing recognition in orthopedics of the use and benefit of SMART technology. SMART stands for Self-Monitoring Analysis and Re $\neg$ porting Technology. Recent clinical research [13] has identified the value and benefits of wearable technology for various populartions raising the question as to whether there may be beneficial use with individuals in treatment for orthopedic related conditions. Wear $\neg$ able SMART technology employs digital technologies to collect health data from individuals in one location, such as a patient's home, and electronically transmit the information to health $\neg$ care providers in a different location for assessment, monitoring and compliance [4]. The orthopedic devices industry continues to be a promising area in the global medical technology area, and is expected to rank third in sales after cardiology and in-vitro diagnostics in the next decade. The growing number of orthopedic patients are aging and can benefit from SMART technology available for both their orthopedic injury as well as the collateral issues the aging population faces when adjusting to an orthopedic condition.

Clinical orthopedic interventions and procedures that reduce both time and expense are improving orthopedic recovery. These interventions are expected to increase as innovative technologies continue to shape the industry. Intelligent orthopedics, which involves a combination of traditional techniques and SMART technology, will be the future of the industry [5,3]. SMART sensor-enabled technologies and implants have provided realtime information to orthopedic surgeons for positioning and post-operative evaluation for better patient care throughout the treatment pathway. These implants have the potential to reduce periprosthetic infection, which is a growing problem in orthopedic practice. Sensor-enabled technologies have given health care providers a choice of unique, cost-effective products. Orthopedic patients will benefit as well from remote monitoring types of technology to aid them in having quick access to emer $\neg$ gency networks. These devices can help foster a safe and indepen $\neg$ dent living environment. Personal wearable smart technology devices are al $\neg$ ready available and the subject of research beneficial to a growing geriatric population. Among these is the Apple "iWatch" which of $\neg$ fers close to a dozen different sensors, tracking the wearers' health and fitness metrics in terms of steps taken, calories burned, blood glucose, sleep quality and heart rate. There are several customized services available for families who have a senior family member needing monitoring. Applications to create a "Smart Medical Home" is impacting the care and treatment of orthopedic patients and aid caregivers who may benefit from computer based technology in their homes to provide necessary monitoring of be $\neg$ havior and maintain health and wellness for the rehabilitating orthopedic patient.

Sport Medicine specialists are engaging biomedical professionals in recognizing the value and benefits of utilizing SMART tech $\neg$ nology options in providing clinical care. E-health is a relatively recent term for healthcare practice utilizing technology-based as $\urcorner$ sessment and treatment strategies that have the potential to deliver benefit to a broad array of individuals who experience anxiety related to their medical conditions [6]. SMART technology is a form of synchronous or asynchronous healthcare delivery that may serve as an adjunct to standardized forms of clinical treatment intervention. Contemporary models of medically supervised healthcare in $\neg$ clude integrated clinical models that emphasize multidisciplinary teams treating patients. Mental health professionals are recognized as bringing specialized skills to the multidisciplinary integrated health care teams. In treating the whole person, contemporary care often requires an integrated team approach that addresses both physical and mental health components involving intervention models that require a limited number of appointments that demonstrate mea $\urcorner$ surable improvements in the current managed care environment. Wearable 
SMART technology provides a potential adjunct for use in assessing the therapeutic progress of some behavioral activities of patients.

University research centers should support greater joint efforts of sport medicine clinicians, scientists and engineers from multiple sites internationally to frame our expanding knowledge and evidence based research studies for SMART healthcare on a multidisciplinary level. Neuro-restoration involves lost neurological function due to the traumatic or ischemic damage to the brain and the associาated neurological deficits. SMART technology can contribute to the rehabilitation of orthopedic patients. Within the SMART medical home [7], a computer tracks the location of preprogrammed items such as eyeglasses, coffee cups, or car keys throughout the house so aging person can locate such items when needed. The SMART technology can aid the person in restoring some lost skills due to brain injury. A camera and scanning system can take three-dimensional textured images of one's skin, automat $\neg$ ically checking for carefully watched skin features such as moles or skin irritation. Other trackers are smart monitors that use cameras located around the home, the personal item. When a picture of the sought-after item is touched on a monitor, the system pinpoints where the item was last recorded, an especially helpful tool for aging persons suffering from forgetfulness brought on by some diseases. Gait Monitors have been developed to track the way the aging person ambulates on various surfaces and in different locations. The system's computers make comparisons over time, checking for any indications of shuffling or limping that may be the precur $\urcorner$ sor to a stroke or for the trembling that may indicate Parkinson's disease. By identifying these ailments early, the hope is that a dis $\urcorner$ ease's full effects can be prevented or ameliorated. Utilization of SMART technology along with an effective diet and exercise program can make all the difference for these patients in achieving improved and recommended health goals in $\neg$ cluding weight, diet, sleep, exercise, self-esteem and an improved quality of life. The use of wearable technology devices like the popular Fitbit [8] has become an enormously popular health \& fitness gauge for about a decade. Health and wellness prone individuals' benefit from self-tracking using such a device as they provide a variety of self-monitoring information and biomarkers.

Within the rehabilitation process the development of the TracPatch is an effective SMART medical device worn by the patient that facilitates healthy post-surgical healing by monitoring range of motion, exercise compliance, and ambulation [5]. TracPatch may be used for both pre and post-op patient monitoring, engagement and critical data analytics. Such wearable devices are easily used by patients as they adhere to the skin above and below the knee. The TracPatch dashboard offers the healthcare provider anytime, anywhere data assessing the patient's recovery. Such technology facilitates for immediate care adjustments that can improve efficiency in the recovery process. With Bluetooth connectivity allows the device to be paired with any smartphone and the HIPAA compliant TracPatch cloud allows for unlimited data collection and storage. The SMART wearable devices are applied before total knee surgery to determine a patient's baseline activity levels, and then again post-surgery to allow the patient and surgeon to stay connected by monitoring activity, pain levels, range of motion and physical therapy progress.

Multiple research efforts have used different terminology to capture the essence of e-health and the overall language used to describe the benefits of wearable SMART technologies. There is clear evidence that the utility and benefits of such technology is being established and beneficial to aging individuals [4]. SMART technology advances for improving healthcare delivery, wearable appliances that form an integral part of our personal life have emerged. The use of 21st century technology is shaping the delivery of biomedical clinical care for orthopedic patients. On the positive side, tech $\neg$ nology's integration with specific counseling skills is inevitable and show favorable potential to assist with improved patient self-management. Because of the early investigations into this modality, single case study design has served as a pilot with the realization that there are a number of limitations. Lacking are certain models or analysis that might include a multiple subject design model and randomization to assess the ef $\neg$ fectiveness of multiple models of the wearable technology. What it represents is an introduction to the use of SMART wearable tech $n$ nology in monitoring and promoting self-management among aging orthopedic patients who experience anxiety related to medical condition that could benefit from life style changes [9].

SMART technology incorporated into the collaborative efforts of orthopedic science and practice clinicians provides a significant pathway with the use of SMART technology in self-monitoring compliance, health and behavior change with a patient mo $\neg$ tivated to utilize smart technology in complying with prescribed clinical treatment continues to contribute to rehabilitation and recovery. Employing available SMART technology for both pre and post-op patient monitoring, engagement and critical data analytics offers both the orthopedic provider and the orthopedic patient improved healthcare efficiency in the treatment and recovery process for orthopedic injury and repair.

\section{References}

1. Bashshur RL, Howell JD, Krupinski KA, Harms KM, Bashshur N, et al. (2016) The Empirical Foundations of Telemedicine Interventions in Primary Care. Telemedicine \& e-health 22(5): 342-375.

2. Van Gaalen JB, Trejos AL, Nikolov HN, Todor G Ivanov, Steven I Pollman (2016) Versatile smart hip implant technology using 3D metal printing 2016. IEEE International Symposium on Circuits and Systems (ISCAS) pp: 2731-2734.

3. O Connor C, Kiourti A (2017) Wireless sensors for smart orthopedic implants. Journal Bio Tribo Corros 3: 20.

4. Miller TW, Kraus RF, Standafer V (2017) SMART Technology: Redefining Geriatric Health Care. Int J Geriatr Gerontol 4(1): 1-7.

5. Andreu-Perez J, Leff DR, Ip HM, Yang GZ (2015) From wearable sensors to smart implants-toward pervasive and personalized healthcare. IEEE Trans Biomed Eng 62(12): 2750-2762. 
6. Allard M, Husky M, Catheline G, Pelletier A, Dilharreguy B, et al. (2014) Mobile technologies in the early detection of cognitive decline 9(12): e112197.

7. Miller TW, Wood J (2012) Telepractice: A 21st Century Model of Health Care Delivery. In: Guah, Matthew (Edt.), Healthcare Delivery: Reform and New Technologies. Information Science Publishers, New York, USA.
8. Chan M, Esteve D, Fourniols JY, Escriba C, Campo E (2012) Smart 6 systems: Current status and future challenges. Artificial Intelligence in Medicine 56(3): 137-156

9. De Lucia PR, Harold SA, Tang YY (2013) Innovation in technology-3. Psychotherapy through human factors/ergonomics: Toward a collaborative approach. Journal of Contemporary Psychotherapy 43: 253-260.

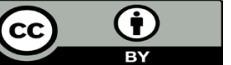

This work is licensed under Creative Commons Attribution 4.0 License

To Submit Your Article Click Here:

Submit Article

DOI: $10.32474 / O S M O A J .2019 .02 .000150$

$\begin{gathered}\text { Orthopedics and Sports Medicine } \\ \text { Open Access Journal }\end{gathered}$
Assets of Publishing with us
- Global archiving of articles
- Immediate, unrestricted online access
- Rigorous Peer Review Process
- Authors Retain Copyrights
- Unique DOI for all articles

